\title{
COMMISSION 37: STAR CLUSTERS AND ASSOCIATIONS
} (AMAS STELLAIRES ET ASSOCIATIONS)

\author{
PRESIDENT: Gary S. Da Costa \\ VICE-PRESIDENT: Georges Meylan \\ ORGANIZING COMMITTEE: S. Aarseth, R. Buonanno, R. Cannon, \\ V. Castellani, K. Cudworth, C. Lada \& A. Sarajedini
}

\section{INTRODUCTION}

With the exception of the activities associated with the XXIII IAU General Assembly in Kyoto, Japan, in August 1997, the report period (July 1, 1996 through June 30, 1999) has been a relatively quiet one for Commission 37 . Commission activities have been restricted primarily to the consideration of proposals for IAU Symposia and Colloquia together with some activity related to cluster nomenclature issues. At the General Assembly the commission was involved in either supporting or co-supporting four Joint Discussion sessions and one of the accompanying Symposia. Eighteen new members were added to the commission, increasing membership by just under $10 \%$.

Other commission activities during the report period included the development of a Commission 37 Web site (accessible through the IAU Web page at www.iau.org). This site lists the commission organizing committee members and their e-mail addresses as well as listing meetings sponsored or co-sponsored by the commission. The web page also has links to two databases of interest to commission members. The first is the Open Clusters Database maintained by Dr. J.-C. Mermilliod at the Institute of Astronomy of the University of Lausanne. The web address for this database is "obswww.unige.ch/webda". The second database is the Catalogue of Milky Way Globular Cluster Parameters (Harris 1996) maintained by Dr. W. E. Harris at McMaster University in Canada. The web address for this catalogue is "physun.physics.mcmaster.ca/Globular.html".

The remainder of this report is devoted to a brief overview of some of the scientific highlights of star cluster research published during the report period. Such compilations are inevitably biased by the interests of the compiler, so I apologize in advance to anyone whose work is worthy of mention here but which I have neglected to include.

\section{RESEARCH HIGHLIGHTS}

\subsection{Globular Clusters}

Within the report period there has been an enormous amount of globular cluster related research published, much of it resulting from Hubble Space Telescope observations. While globular clusters may appear to be simple systems, as we study them in greater detail an increasing degree of complexity becomes evident. That statement is no better illustrated than by noting the diversity of horizontal branch morphologies revealed by a number of recent studies. For example, Rich et al. (1997) have shown that the metal-rich globular clusters NGC 6388 and NGC 6441, in addition to the expected red horizontal branch (HB) stars, also possess prominent blue HB extensions. On the other hand, two other metal-rich clusters (47 Tuc and NGC 5927) studied in the same way, lack these blue HB stars. As the authors themselves admit, the cause of this phenomenon remains uncertain. Similarly, Sosin et al. (1997) have revealed that the HB morphology of the cluster NGC 2808, already known 
to bimodal, has in fact a long blue tail with two distinct gaps, indicating the possibility of four distinct groups of $\mathrm{HB}$ stars in the one cluster. A multi-mode $\mathrm{HB}$ mass distribution, of unknown origin, is required to explain this distribution (e.g. Catelan et al. 1998). As a further complication, Behr et al. (1999) have used high resolution spectra to reveal enormous anomalies (e.g. Fe abundances 2 orders of magnitude above the canonical cluster metallicity!) in the atmospheres of blue HB stars in the globular cluster M13. While these anomalies are presumably the result of gravitational settling/radiative levitation processes, it is possible that they could also affect cluster HB morphology.

Ages - Absolute and Relative. One of the most fundamental cosmological tests is the comparison of the age of the oldest stars, as measured from globular cluster main sequence turnoffs, with the age of the Universe as inferred from cosmological parameters. The absolute age of a globular cluster is, of course, strongly dependent on the distance assumed for the cluster. During the report period accurate parallaxes for nearby metal-poor subdwarfs became available from the Hipparcos catalogue. This led, inter alia, to a revaluation of the distances to globular clusters. The results are discussed in Chaboyer et al. (1998, see also Pont et al. 1998) who reviewed five independent techniques used to set the distance scale for globular clusters. Their results imply that globular clusters are generally further away than previously assumed, and thus younger in age. Chaboyer et al.'s best estimate for the mean age of the oldest Galactic globular clusters is $11.5 \pm 1.3 \mathrm{Gyr}$. This lower limit on the age of the Universe is consistent with recent determinations of the Hubble constant.

Relative ages for globular clusters, however, especially for clusters with similar abundances, are generally easier to measure than absolute ages. Here too there has been important progress. HST/WFPC2 data have allowed accurate comparison of the main sequence turnoffs of a metal-poor globular cluster in the outer Galactic halo (NGC 2419, Harris et al. 1997), metal-poor globular clusters in the Large Magellanic Cloud (Olsen et al. 1998) and metal-poor globular clusters in the Fornax dwarf spheroidal galaxy (Buonanno et al. 1998), with metal-poor globular clusters in the solar neighbourhood. These studies have revealed the remarkable result that, regardless of the subsequent star formation histories, the initial epoch of star formation was well synchronized (to within $1 \mathrm{Gyr}$ ) among all the components of the proto-Galactic halo, which may have been distributed over a volume at least $\sim 100 \mathrm{kpc}$ in radius. HST/WFPC2 photometry has also allowed direct confirmation, via main sequence turnoff photometry, of the long-held view that the low luminosity globular clusters in the outer Galactic halo with red HB morphologies are younger than the classic nearby halo globular clusters. Specifically, Stetson et al. (1999) find that the red HB morphology outer halo clusters Pal 3, Pal 4 and Eridanus are younger by 1.5-2 Gyr compared to inner halo counterparts, such as M3 and M5, provided there are no chemical abundance ratio differences between the inner and outer halo objects.

Abundance Anomalies. One of the long standing puzzles of globular cluster research is the origin of the star-to-star abundance anomalies found in many globular clusters. During the report period this puzzle has remained unsolved but new insight has been provided from both the observational (e.g. Kraft et al. 1998 and references therein) and theoretical (e.g. Cavallo, Sweigart \& Bell 1998, Denissenkov et al. 1998) point-of-view. Highlights include a report of spectroscopic evidence for small star-to-star variations in the abundances of the Fe-peak elements among the red giants in the metal-poor globular cluster M92 (Langer et al. 1998). Specifically, Langer et al. (1998) find that one M92 red giant has an average abundance of the Fe-peak elements that is $0.18 \mathrm{dex}$ higher than the value for two other red giants. All three stars have very similar temperatures and gravities. Previously, starto-star abundance variations in this cluster had been restricted to the elements $\mathrm{C}, \mathrm{N}, \mathrm{O}$, $\mathrm{Na}, \mathrm{Mg}$ and Al. Similarly, Sneden et al. (1997) have discovered significant (range $\sim 0.6$ dex) variations in the $[\mathrm{Eu} / \mathrm{Fe}]$ and $[\mathrm{Ba} / \mathrm{Fe}]$ abundance ratios among the red giants in the metal-poor cluster M15. Such abundance ratio variations had previously been seen only in the cluster $\omega$ Cen. Interestingly, the $[\mathrm{Eu} / \mathrm{Fe}]$ and $[\mathrm{Ba} / \mathrm{Fe}]$ variations in the M15 red giants 
appear to be independent of the ubiquitous variations in the elements $\mathrm{C}, \mathrm{N}, \mathrm{O}, \mathrm{Na}, \mathrm{Mg}$ and $\mathrm{Al}$, which are seen in this and many other globular clusters.

Recent work (Smith, Shetrone \& Keane 1999, Kraft et al. 1999) has now added Li to the list of elements that vary from star-to-star among globular cluster red giants. All low mass red giants should have destroyed or diluted their photospheric Li abundances to very low levels, so the detection of $\mathrm{Li}$ features in the spectrum of any globular cluster red giant comes as a surprise. Smith et al. (1999) find $\log \epsilon(\mathrm{Li})=1.2 \pm 0.2$ for one red giant in the globular cluster NGC 362. This star does not show any $\mathrm{C}, \mathrm{N}, \mathrm{O}, \mathrm{Na}, \mathrm{Mg}$ or Al abundance anomalies. Similarly, Kraft et al. (1999) find $\log \epsilon(\mathrm{Li}) \approx 3.0$ for one red giant in M3. But, in contrast to the NGC 362 Li-rich red giant, this M3 star also shows the $\mathrm{C}, \mathrm{N}, \mathrm{O}, \mathrm{Na}, \mathrm{Mg}$ and $\mathrm{Al}$ abundance anomalies seen in (some) other $\mathrm{M} 3$ red giants.

Most studies of globular cluster abundance anomalies have been restricted to the brighter stars in the cluster. With the advent of multi-object spectrographs, however, stars on the main sequence in globular clusters are now being increasingly studied. Cohen (1999) reports that spectra of 79 stars just below the main sequence turnoff in the cluster M71 reveal significant star-to-star variations in the strengths of CH (G-band) and CN (band at $\lambda 3885 \AA$ ) features. To first order the band strength variations are bimodal and anti-correlated with the numbers of $\mathrm{CN}$-strong/CH-weak and $\mathrm{CN}$-weak/CH-strong stars being approximately equal. These results are similar to those found by Cannon et al. (1998) for main sequence stars in $\mathbf{4 7}$ Tuc and they remove any possibility that 47 Tuc could be considered unique or a special case.

Finally, Norris et al. (1997) discuss correlations between kinematics and abundance in the chemically inhomogenous cluster $\omega$ Cen. They find that the stars in the $20 \%$ metalrich tail of the abundance distribution, as well as being more centrally concentrated, have a lower velocity dispersion than the stars in the $80 \%$ metal-poorer component. However, surprisingly, the metal-rich component shows no evidence for the systematic rotation readily seen in the metal-poorer component. This rules out using simple dissipative collapse models to connect the kinematics and abundances.

Other Results. Three other subjects deserve some comment. First, Dinescu, Girard \& van Altena (1999) have complied a catalogue of absolute proper motions for 38 globular clusters, including 15 southern clusters measured as part of their own program (see references in Dinescu et al. 1999). These motions were used as a basis for orbit integrations using models of the Galaxy's potential. Three metal-poor clusters (NGC 6254, NGC 6626 and NGC 6752) were found to have orbits similar to those of the metal-rich "disk" globular clusters. This suggests there is a low metallicity component in the Galaxy's thick disk population. If this is the case, then the age of these three clusters indicates that the formation of the disk overlapped with that of the halo. Further, the globular clusters classified as "young halo" or "red horizontal branch" were found to have a radially anisotropic velocity distribution and highly eccentric orbits of high total energy and large apocentric radius. This suggests that these clusters represent an accreted component in the Galaxy's halo.

Second, deep HST/WFPC2 observations have been used to identify the white dwarf populations of three nearby globular clusters. Cool, Piotto, \& King (1996) have delineated a sequence of $\sim 40$ white dwarfs with $\mathrm{M}_{V} \approx 10.5-14$ in NGC 6397. The brighter stars in this sequence match well theoretical cooling sequences for white dwarfs with masses of $0.55 \pm 0.05 \mathrm{M}_{\odot}$. Their numbers are also consistent with the expected rate of white dwarf formation given the current evolutionary rate past the red giant branch tip. Similarly, Richer et al. (1997) have identified a large population white dwarfs in M4. The location of these white dwarfs in the cluster colour-magnitude (c-m) diagram corresponds to a mean mass of $0.51 \pm 0.03 \mathrm{M}_{\odot}$, and again supports current white dwarf cooling theory. At the detection limit of the data the white dwarf luminosity corresponds to an age of $\sim 9 \mathrm{Gyr}$, providing a lower limit on the age of this cluster. Finally, Renzini et al. (1996) have detected the white dwarf sequence in NGC 6752 and have compared it to a sample of local white 
dwarfs with known trigonometric parallaxes. This comparison yields a distance modulus for the cluster directly, with an uncertainty of less that \pm 0.1 mag.

Third, King et al. (1998) have used second epoch HST/WFPC2 observations to measure proper motions accurate enough to separate field stars from low luminosity members of NGC 6397. This technique has allowed the cluster main sequence luminosity function to be extended to magnitudes $\left(\mathrm{M}_{I} \approx 12.5\right)$ that approach the hydrogen burning limit which, for the cluster's metallicity, corresponds to a mass of less than $0.1 \mathrm{M}_{\odot}$. Theoretical models of low mass stars predict a rapid drop in the main sequence luminosity function as the hydrogen burning limit is approached, and this prediction is consistent with the observations.

\subsection{Open Clusters}

One of the most important papers for star cluster research published in the report period is the detailed study of the distance, structure, dynamics and age of the Hyades cluster by Perryman et al. (1998). These authors have used absolute parallaxes from the Hipparcos Catalogue to determine individual distances to Hyades members, allowing a derivation of the 3D structure of the cluster and an accurate c-m diagram. Among the results are a derivation of the cluster belium abundance $(\mathrm{Y}=0.26 \pm 0.02)$ and a precise distance determination for the cluster centre-of-mass $\left((\mathrm{m}-\mathrm{M})_{0}=3.33 \pm 0.01 \mathrm{mag}\right)$.

A fundamental reason for studying open clusters is the ability of observed cluster c-m diagrams to constrain theoretical models of stellar evolution. In this respect the fact that open clusters range in age from a few $\mathrm{Myr}$ to a few $\mathrm{Gyr}$ is particularly important because it permits comparison with theory over a broad range of masses. In the report period there has been a sizeable increase in the number of open cluster c-m diagrams published, both for well known clusters and for previously unstudied objects. This is primarily due to the increasing use of large format CCD detectors. An example of work of this type is the paper of Rosvick \& VandenBerg (1998) which presents new photometry for the populous open cluster NGC 6819. The authors find that the morphology of the cluster c-m diagram in the vicinity of the main sequence turnoff can be matched by theory only if a significant amount of convective core overshoot is used in the evolutionary models. Standard non-overshoot models fail by a wide margin to explain the observations. Similar results were found by Nordström et al. (1997) in their detailed study of NGC 3680. While a general requirement for core overshoot has been known for some time, these papers illustrate the insight that can be gained through the interaction of theory and observation. A similar need for core overshoot models to fit c-m diagram features has also been found for NGC 7789. This cluster has been studied both kinematically and photometrically by Gim et al. (1998a, b).

Another open cluster of significance that has been recently discussed anew is NGC 6791. It is an important object because its old age and high metallicity, now well established as $[\mathrm{Fe} / \mathrm{H}]=+0.4 \pm 0.1$ (Peterson \& Green 1998), mean that it can serve as a template for the populations expected in the bulges of elliptical and spiral galaxies. Chaboyer, Green \& Leibert (1999) have calculated an extensive grid of metal-rich isochrones for this cluster utilizing the latest available input physics. They then compared these isochrones with available observations of the cluster. The best fitting isochrone, which assumes $[\mathrm{Fe} / \mathrm{H}]=$ +0.4 , scaled solar abundance ratios and $\Delta Y / \Delta Z=2$ (i.e. $Y=0.31$ ), gives an excellent fit at all points along the major sequences in the cluster c-m diagrams. The derived age is 8.0 Gyr with an uncertainty of less than $1 \mathrm{Gyr}$. This age is consistent with the reduced ages now being derived for all clusters (old open and globular) as a result of comparisons with the newest isochrones, colour calibrations and Hipparcos distances.

A further example of progress in comparing stellar evolution theory with observation is the recent results of Richer et al. (1998). These authors have used deep wide field CCD imaging of M67 to determine not only the location of the cluster main sequence down to stars as faint as $\mathrm{M}_{V} \approx 13.5$ (mass $\approx 0.15 \mathrm{M}_{\odot}$ ) but also to establish the white dwarf cooling sequence in this cluster. The observed white dwarf sequence follows quite closely a theoretical cooling sequence and the termination magnitude, $M_{V} \approx 14.6$, indicates a white 
dwarf cooling age for the cluster of $4.3 \mathrm{Gyr}$. This agrees remarkably well with the main sequence turnoff age, determined by isochrone fits, of $4.0 \mathrm{Gyr}$.

A second fundamental aspect of open cluster research is the determination of main sequence luminosity functions, which can yield information on either the dynamical evolution of the cluster or on the cluster initial mass function. For example, von Hippel \& Sarajedini (1998) have used very deep imaging observations to investigate the main sequence luminosity function (LF) in the central regions of NGC 188. They find that unlike the solar neighbourhood LF, and unlike the LFs of dynamically unevolved regions of open and globular clusters, the NGC 188 central region LF peaks at the surprisingly bright value of $\mathrm{M}_{I} \approx 3$ to 4 . To explain this result von Hippel \& Sarajedini (1998) suggest that NGC 188 is highly dynamically evolved and that the low luminosity stars missing from the central regions of the cluster are either located in the cluster outskirts or have escaped the cluster entirely. Similarly, Nordström et al. (1997) have shown that NGC 3680 lacks cluster stars fainter than $\mathrm{M}_{V} \approx 4$ while the fraction of binaries among cluster members is significantly higher than in the field. Evidently this cluster is also highly dynamically evolved with most of the original cluster low mass stars again either in extended halo or lost completely from the cluster. Other studies of the dynamics of open clusters include the work of Raboud \& Mermilliod (1998a,b).

The report period has also seen the confirmation of the existence of Brown Dwarfs in open clusters such as the Pleiades. Brown Dwarf searches in the Pleiades have been particularly successful because this cluster offers the ideal combination of young age, proximity, high metallicity, low reddening, richness and compactness (see Martín et al. 1998 and the references therein). Recent low resolution spectroscopic studies of some of these Pleiades Brown Dwarf candidates have revealed the first L-type Brown Dwarf in this cluster (Martín et al. 1998). The spectra of L-type (as distinct from the somewhat hotter M-type) Brown Dwarfs lack strong TiO bands and show instead metal-hydride (e.g. $\mathrm{CaH}, \mathrm{CrH}$ ) bands. The existence of this star in the Pleiades shows that the cluster initial mass function extends down to at least $\sim 0.035 \mathrm{M}_{\odot}$ (Martín et al. 1998).

\subsection{Associations}

In the past year multi-wavelength (X-ray, optical, IR) observations have been used to establish the existence of two very nearby associations of young stars, both of which are unexpectedly far from any known molecular clouds. The first of these is the TW Hydrae association. Webb et al. (1999) have shown that there are at least eleven young star systems, containing at least 19 stars as well as a probable brown dwarf, in the vicinity of the isolated classical T Tauri star TW Hyr. This association is the nearest region of recent star formation yet it is completely isolated from any known molecular cloud. With an age of $\sim 10 \mathrm{Myr}$ and a distance of $\sim 50 \mathrm{pc}$ this association represents an excellent place to study circumstellar disk dissipation and the formation of planets and brown dwarfs. For example, the colour and brightness of the brown dwarf candidate identified by Webb et al. (1999) suggest it has a mass of $\sim 20 \mathrm{M}_{J u p}$.

Similarly, Mamajek et al. (1999) report the discovery of a second nearby $(\mathrm{d} \approx 100 \mathrm{pc}$ ) association of young stars - the $\eta$ Chamaeleontis cluster. This cluster contains stars with ages from approximately 2 to $18 \mathrm{Myr}$, and, like the TW Hya association, it is far from any significant molecular cloud. The cluster, however, appears to share proper motions with other young stars in the Chamaeleon region and it may be a compact extension of the Sco-Cen OB association (Mamajek et al. 1999).

Dr. Gary S. Da Costa

President of the Commission

\section{References}

Behr, B. B., Cohen, J. G., McCarthy, J. K., \& Djorgovski, S. G. 1999, ApJ, 517, L135 
Bounanno, R., Corsi, C. E., Zinn, R., Fusi Pecci, F., Hardy, E., \& Suntzeff, N. B. 1998, ApJ, 501, L33

Cannon, R. D., Croke, B. F. W., Bell, R. A., Hesser, J. E., \& Stathakis, R. A. 1998, MNRAS, 298, 601

Catelan, M., Borissova, J., Sweigart, A. V., \& Spassova, N. 1998, ApJ, 494, 265

Cavallo, R. M., Sweigart, A. V., \& Bell, R. A. 1998, ApJ, 492, 575

Chaboyer, B., Demarque, P., Kernan, P. J., \& Krauss, L. M. 1998, ApJ, 494, 96

Chaboyer, B., Green, E. M., \& Liebert, J. 1999, AJ, 117, 1360

Cohen, J. G. 1999, AJ, 117, 2434

Cool, A. M., Piotto, G., \& King, I. R. 1996, ApJ, 655

Denissenkov, P. A., Da Costa, G. S., Norris, J. E., \& Weiss, A. 1998, A\&A, 333, 926

Dinescu, D., Girard, T. M., \& van Altena, W. F. 1999, AJ, 117, 1792

Gim, M., Hesser, J. E., McClure, R. D., \& Stetson, P. B. 1998a, PASP, 110, 1172

Gim, M., VandenBerg, D. A., Stetson, P. B., Hesser, J. E., \& Zureck, D. R. 1998b, PASP, 110,1318

Harris, W. E. 1996, AJ, 112, 1487

Harris, W. E., et al. 1997, AJ, 114, 1030

von Hippel, T., \& Sarajedini, A. 1998, AJ, 116, 1789

King, I. R., Anderson, J., Cool, A. M., \& Piotto, G. 1998, ApJ, 492, L37

Kraft, R. P., Peterson, R. C., Guhathakurta, P., Sneden, C., Fulbright, J. P., \& Langer, G. E. 1999, ApJ, 518, L53

Kraft, R. P., Sneden, C., Smith, G. H., Shetrone, M. D., \& Fulbright, J. 1998, AJ, 115, 1500

Langer, G. E., Fischer, D., Sneden, C., \& Bolte, M. 1998, AJ, 115, 685

Mamajek, E. E., Lawson, W. A., \& Feigelson, E. D. 1999, ApJ, 516, L77

Martín, E. L., Basri, G., Zapatero-Osorio, M. R., Rebolo, R., \& García López 1998, ApJ, $507, \mathrm{~L} 41$

Nordström, B., Andersen, J., \& Andersen, M. I., 1997, A\&A, 322, 460

Norris, J. E., Freeman, K. C., Mayor, M., \& Seitzer, P. 1997, ApJ, 487, L187

Olsen, K. A. G., Hodge, P. W., Mateo, M., Olszewski, E. W., Schommer, R. A., Suntzeff, N. B., \& Walker, A. R. 1998, MNRAS, 300, 665

Perryman, M. A. C., et al. 1998, A\&A, 331, 181

Peterson, R. C., \& Green, E. M. 1998, ApJ, 502, L39

Pont, F., Mayor, M., Turon, C., \& VandenBerg, D. A. 1998, A\&A, 329, 87

Raboud, D., \& Mermilliod, J.-C. 1998a, A\&A, 329, 101

Raboud, D., \& Mermilliod, J.-C. 1998a, A\&A, 333, 897

Renzini, A., et al. ApJ, 465, L23

Rich, R. M., et al. 1997, ApJ, 484, L25

Richer, H. B., et al. 1997, ApJ, 484, 741

Richer, H. B., Fahlman, G. G., Rosvick, J., \& Ibata, R. 1998, ApJ, 504, L91

Rosvick, J. M., \& VandenBerg, D. A. 1998, AJ, 115, 1516

Sosin, C., et al. 1997, ApJ, 480, L35

Smith, V. V., Shetrone, M. D., \& Keane, M. J. 1999, ApJ, 516, L73

Sneden, C., Kraft, R. P., Shetrone, M. D., Smith, G. H., Langer, G. E., \& Prosser, C. F. 1997, AJ, 114, 1964

Stetson, P. B., et al. 1999, AJ, 117, 247

Webb, R. A., Zuckerman, B., Platais, I., Patience, J., White, R. J., Schwartz, M. J., \& McCarthy, C. 1999, ApJ, 512, L63 
